Biosight 2021; 02(02): 13-21

\title{
The Effect of Fenugreek Seeds (Trigonella foenum-Gracecum) Supplementation on Glycemic Status, Androgens, And Lipid Profile in Letrozole Induced Polycystic Ovarian Syndrome (PCOS) Model
}

\author{
Bushra Manzoor Lodhi* and Uzma Firdous \\ Department of Physiology, University of Karachi, Karachi, Pakistan
}

Received: April 16, 2021 Accepted: September 7, 2021 doi: $10.46568 / \mathrm{bios}, \mathrm{v} 2 \mathrm{i} 1.35$

\begin{abstract}
:
Introduction: PCOS the most common endocrinopathy of reproductive-aged females is hallmarked by characteristics of polycystic ovary syndrome and biological hyperandrogenism and hyperinsulinism. Fenugreek seeds have proven to be beneficial in the treatment of PCOS because of their antiandrogenic and anti-hyperglycaemic effects. The present study was aimed to evaluate the effect of aqueous extract of fenugreek seed in PCOS rats. Materials and method: Twenty-four female Wistar rats were divided into two groups: Group 1 served as a (control group, $\mathrm{n}=10)$ received saline $(0.9 \% \mathrm{NaCl})$ while Group 2 (PCOS control group, $\mathrm{n}=30)$ received letrozole $(1 \mathrm{mg} / \mathrm{kg})$ to induce PCOS. After 21 days, PCOSinduced rats were divided into two groups. One group was treated with the aqueous extract of fenugreek seeds and another group was treated with metformin ( $300 \mathrm{mg} / \mathrm{Kg}$ ) for 21 consecutive days while the control group continued to receive saline. At the end of the experiment ovaries were removed for histological examination and blood collected for the estimation of Insulin, Testosterone, and Lipid profile. Result: Compared with the control group, Letrozole administration in rats exhibited significant alterations in serum testosterone, insulin activity, glucose and Lipid profile $(\mathrm{P}<0.001)$. Acyclicity of the estrus cycle with cysts were also detected. In Fenugreek and metformin groups restoration of normal ovarian stroma, normalization of estrous cycle with reduced levels of testosterone $(\mathrm{P}<0.001)$, insulin $(\mathrm{P}<0.001)$, glucose $(\mathrm{P}<0.001)$, and cholesterol $(\mathrm{P}<0.0001)$ were observed. Discussion: These findings suggest that aqueous extract of fenugreek showed better amelioration in the regulation of impaired insulin resistance, hyperandrogenaemia, and ovarian dysfunction in PCOS.
\end{abstract}

Keywords: PCOS, Fenugreek, Letrozole, Hirsutism, Hyperandrogenism

*Correspondence: Bushra Manzoor Lodhi, Department of Physiology, University of Karachi, Karachi, Pakistan, Tel: 03152487959, Email: lodhibushra4@gmail.com

\section{Introduction}

Polycystic ovary (Stein Leventhal Syndrome) is one of the major endocrine and metabolic disorders of women affecting approximately $4-10 \%$ women of reproductive women [1, 2]. It is characterized by hyperandrogenism, multiple small ovarian cysts, irregular menstrual cycles, and anovulation. The elevated level of testosterone leads to inhibition of aromatization of androgens which ultimately increased the androgen levels. Hirsutism, acne, weight gain, miscarriage, infertility, high anxiety levels, depression are also associated with PCOS [3]. The exact etiology of this syndrome is still unidentified and it remains the most perplexing and multifactorial disorder of the female reproductive system.

Many therapies have been used to manage the signs and symptoms of PCOS. Metformin has been used as the primary choice for the derangements caused by PCOS [4]. Metformin is an insulinsensitizing agent act by inhibiting hepatic gluconeogenesis[5]. It regulates the level of insulin and reduces the free testosterone in the blood. Metformin also controls ovarian hyperandrogenism in PCOS. However, long-term administration of metformin produces gastrointestinal side effects [5, 6]. 
Insulin resistance and the associated compensatory hyperinsulinemia are the common manifestations in PCOS women and may act as a core pathogenetic factor in the development of this syndrome. Insulin resistance and hyperandrogenism are interrelated with each other and play a central role in the occurrence and development of PCOS [7].

Due to the high cost of fertility treatments, the use of Complementary and Herbal Medicines (CAMs), an alternate treatment with herbs is increasing globally for the treatment of chronic diseases. Fenugreek (Trigonella foenum-graecum) is a homegrown herb and it has been used as an anti-diabetic and anti hypercholesterolemic agent in both human and animal studies [8]. The active components of the fenugreek extract such as flavonoids, saponins, alkaloids, steroids, and fibers are responsible for the hypoglycemic effects of the fenugreek herb.

This study was designed to evaluate the impact of Fenugreek seeds on the reproductive, androgens, insulin resistance, and lipid parameters in letrozole-induced PCOS. To create a PCOS model in rats, we used letrozole, the third-generation non-steroidal aromatase inhibitor. The aromatase is the key enzyme that converts testosterone and androsterone into estrogen. The aromatase enzyme is inhibited by the letrozole, by its competitive binding and increases ovarian androgens, which results in hyperandrogenism, leading to disturbed estrous cycle, polycystic ovaries, and hirsutism [9].

\section{Materials and Methods}

\section{Preparation of Aqueous Extract of Fenugreek Seeds}

Fenugreek Seeds were purchased from the local market. After collection, cleaned fenugreek seeds were dried and powdered finely with an herbal grinder. 20 grams of powdered fenugreek seeds were boiled in $500 \mathrm{ml}$ of distilled water for 1 hour. The boiled extract was left for a whole night and then filtered making up to $500 \mathrm{ml}$ again with distilled water [10]. The fenugreek aqueous extract can be stored in the refrigerator for further use.

\section{Selection of Animals}

24 female Albino Wistar Rats (nine weeks old, 110-120 gram weight) were purchased from the animal house of Panjwani Medical Centre (PCMD). The rats were placed in an animal house of the Department of Physiology, University of Karachi. The experimental protocols were approved by the Institutional Animal Care and Use Committee and strictly followed guidelines provided by the National Institute of Health $(\mathrm{NIH})$ for the care and use of laboratory animals. All rats were acclimatized for one week by housing in separate cages under standard conditions of temperature $\left(23 \pm 12^{\circ} \mathrm{C}\right)$, a 12 -hour light-dark cycle with free access to water, and a balanced pellet diet. After 1 week of the acclimatization period, the estrous cycle was observed daily with the help of vaginal smearing till the end of the experiment.

\section{Grouping of Animals}

In the first phase, 24 rats were divided into two groups. $1^{\text {st }}$ Group (Control group, $n=6$ ) received saline $(0.9 \% \mathrm{NaCl})$ while the $2^{\text {nd }}$ group (PCOS control group, $\left.\mathrm{n}=18\right)$ received letrozole $1 \mathrm{mg} / \mathrm{kg}$ body weight to induce PCOS. The weight of each rat was taken after every 2 days. After 21 days of treatment, few rats were sacrificed to confirm the presence of PCOS.

In the second phase, the control group continued to receive saline $(0.9 \% \mathrm{NaCl})$ whereas PCOSinduced rats were divided into two equal groups. One group (Group III) was treated with the aqueous extract of fenugreek seeds (1mg/Kg body weight) while the other group (Group IV) was treated with metformin $(300 \mathrm{mg} / \mathrm{Kg}$ body weight) for 21 days. Before sacrificed, all rats were weighed and fasted overnight. After the infusion of anesthesia (Chloral hydrate 10\%), the blood sample was collected from the abdominal aorta and centrifuged at 2500 grams for 10 minutes and stored at $-80^{\circ} \mathrm{C}$. Ovaries were collected and fixed in $10 \%$ formalin for the histological examination.

\section{Preparation of Vaginal Smear}


Vaginal smears were collected by using used cotton bud method to identify the stage of cyclicity from 42 days of age till the end of the experiment. In this method, the cotton bud is dip in normal saline $(0.9 \mathrm{NaCl})$ and injected approximately $10 \mathrm{~mm}$ into the depth of the vagina by rotating at an angle of about $45^{\circ}$. After gently removing the swab, the vaginal secretions were smeared on the clean labeled glass slides. The smeared slides were heat-dried and stained with a $0.1 \%$ crystal violet stain for at least 1 mint followed by quick washing of the slide in distilled water to remove the excess stain on slides [11]. The slide of prepared vaginal smear observed under a light microscope using 10x magnification to analyze the cytological changes in the estrous cycle.

\section{Statistical Analysis}

Statistical evaluations were performed with SPSS software (version 22.0). Data were expressed as mean \pm SD. One-way ANOVA with Tukey test was applied to examine the statistical difference among the groups and $\mathrm{P}<0.05$ was considered to be significant.

\section{Results:}

\section{Mean length of the Estrous Cycle}

The Control group (Group I) showed regular estrus cycles of 4-5 days (Figure 2) which comprised of proestrus, estrus, metestrus, and diestrus (Figure 1A, 1B, 1C, and 1D). Light microscopic analysis showed no structural abnormalities in the control group (Group I) whereas the PCOS induced group (Group II) showed the presence of numerous leukocytes, persistent diestrus phase (figure 1E), and the non-identified phase known as pseudo-diestrus phase (Figure 2). In Group III (fenugreek treated group) and Group IV (metformin-treated group), no changes were observed in the pseudodiestrus phase during the first fifteen days which gradually restored to normal estrous cycle with an increase in metestrus phase.

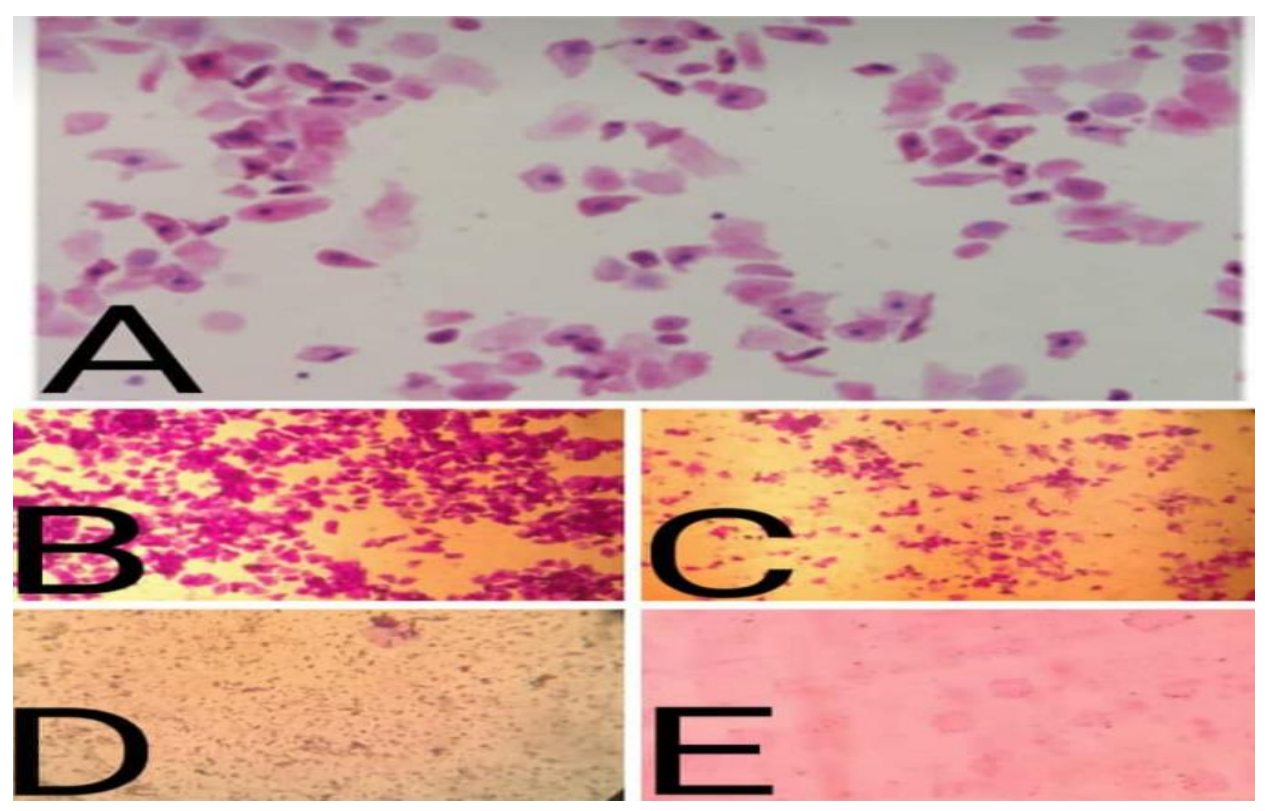

Figure 1. Phases of the estrous cycle of rats: (A) showing normal proestrus phase; (B) represents normal estrus phase; (C) showing normal metestrus phase; (D) representing normal diestrus phase; (E) showing the abnormal phase of the estrous cycle which normally appear in PCOS, also called as the Pseudodiestrus phase.

\section{Changes in Body Weight}

PCOS-induced rats exhibited a significant increase in their body weight concerning the normal control group $(\mathrm{P}<0.01)$. However, treatment with fenugreek and metformin advanced to a significant decrease in their body weight as compared to PCOS induced group $(\mathrm{P}<0.05)$. 


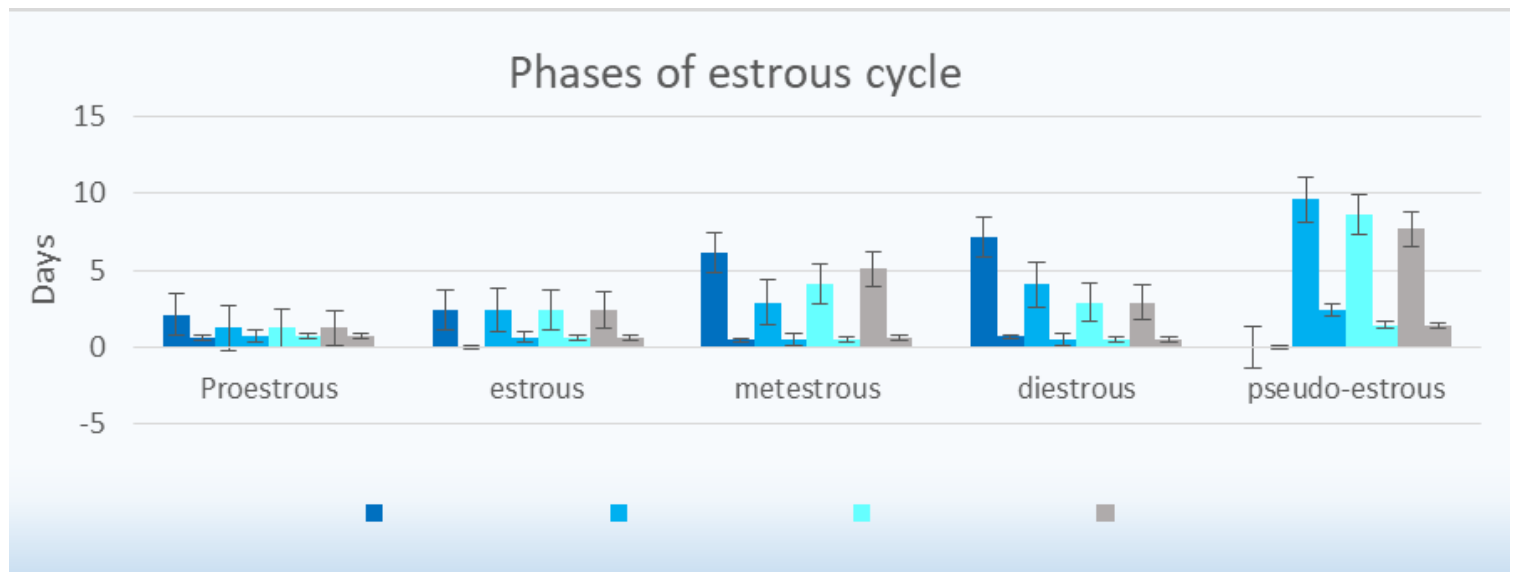

Figure 2. Mean length of estrous cycle phases: Group I showed normal cycle; Group II showed irregular cycles with the increased no of pseudo-diestrus phase; Group III showed marked recovery in estrous cycle phases with the complete disappearance of pseudo-diestrus phase and Group IV as well showed improvement in the estrus phases.

\section{Body weight}

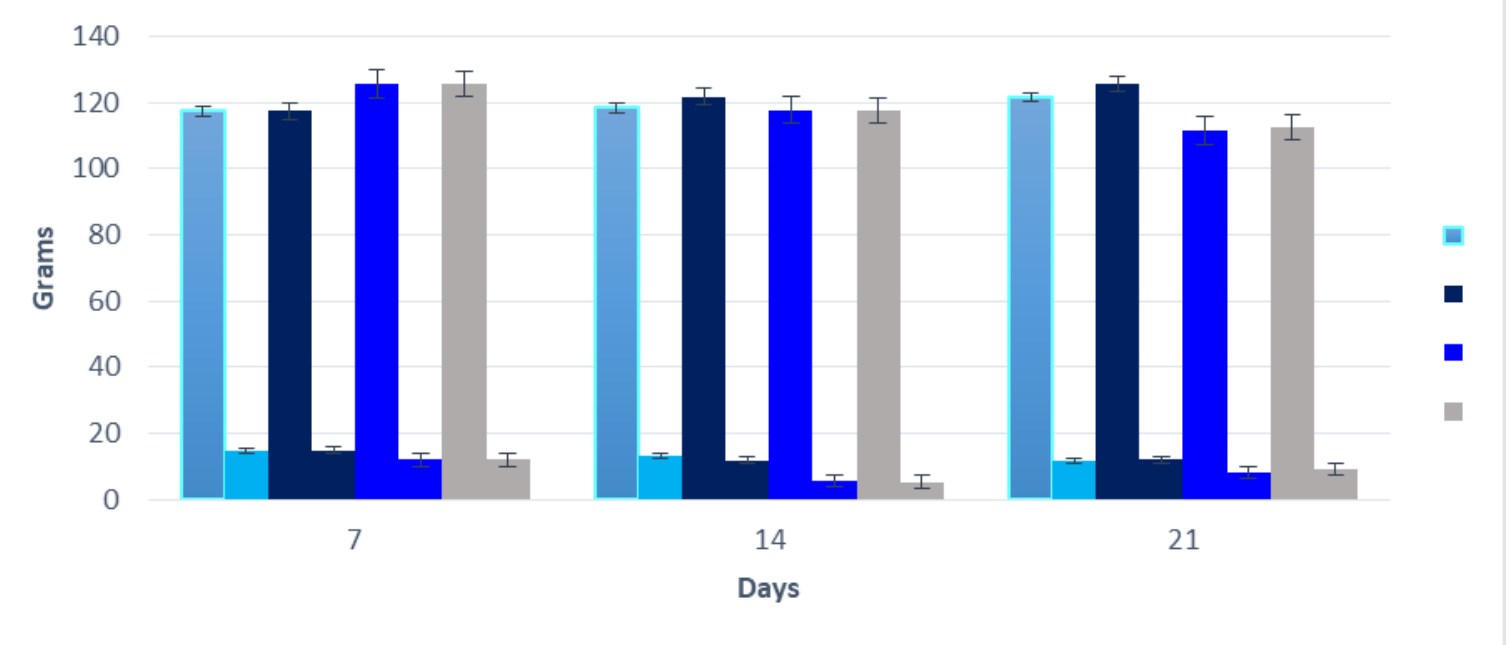

Figure 3 Comparison of body weight in all groups: control group I show significant increase in their weight; PCOS-induced control group II demonstrate increased in their weight; Fenugreek group III show significant decline in the weight and Metformin group IV illustrate decline in their body weight.

\section{Comparison of Endocrine Variables among the Groups}

Table 1 showed that Group II exhibited a highly significant increase in glucose, insulin, and testosterone levels as compared to the control group $(\mathrm{P}<0.001)$. On the other hand, a significant decrease in glucose, insulin, and testosterone levels was observed in the fenugreek and metformintreated group with respect to PCOS induced group $(\mathrm{P}<0.001)$.

Table 1: Comparison of Endocrine variables of rats among the groups

\begin{tabular}{|l|l|l|l|l|}
\hline GROUPS & $\begin{array}{l}\text { Glucose}(\mathrm{mg} / \mathrm{d} \\
1)\end{array}$ & $\begin{array}{l}\text { Insulin } \\
\boldsymbol{\mu U} / \mathrm{ml})\end{array}$ & HOMA- IR & $\begin{array}{l}\text { Testosterone } \\
\text { ng/dl) }\end{array}$ \\
\hline $\begin{array}{l}\text { Normal Control } \\
\text { Group (Group I) }\end{array}$ & $80.8 \pm 3.488$ & $5.45 \pm 0.580$ & $1.05 \pm 0.127$ & $31.85 \pm 0.62$ \\
\hline $\begin{array}{l}\text { PCOS induced } \\
\text { Group (Group II }\end{array}$ & \begin{tabular}{l}
$\# \#$ \\
\hline
\end{tabular} & $\begin{array}{l}9.32 \pm 0.394 \\
\# \#\end{array}$ & $\begin{array}{l}2.398 \pm 0.116 \\
\# \#\end{array}$ & $\begin{array}{l}60.22 \pm 0.932 \\
\# \#\end{array}$ \\
\hline
\end{tabular}


Biosight 2021; 02(02): 13-21

\begin{tabular}{|ll|l|l|l|l|}
\hline $\begin{array}{l}\text { Fenugreek } \\
\text { (Group III) }\end{array}$ & Group & $91.5 \pm 6.8920$ & $6.60 \pm 0.454$ & $1.398 \pm 0.114$ & $23.47 \pm 0.550$ \\
& & $\#$ & $\#$ & $\#$ & $\#$ \\
& $\wedge$ & $\wedge$ & $\wedge$ & $\wedge$ \\
\hline $\begin{array}{l}\text { Metformin } \\
\text { (Group IV) }\end{array}$ & Group & $\begin{array}{l}81.83 \pm 3.488 \\
\#^{*}\end{array}$ & $\begin{array}{l}5.00 \pm .1633 \\
\# *\end{array}$ & $\begin{array}{l}1.206 \pm 0.049 \\
\# *\end{array}$ & $\begin{array}{l}18.75 \pm 0.506 \\
\#\end{array}$ \\
\hline
\end{tabular}

Values are presented as mean \pm S.D, applied one-way ANOVA followed by Tukey test

$(\mathrm{n}=6), \mathrm{ns}=$ not significant

$\mathrm{P}<0.001 \#$ significantly different from control group.

$\mathrm{P}>0.05$ \#\#significantly different from control group.

$\mathrm{P}<0.001^{\wedge}$ significantly different from PCOS treated with Fenugreek.

$\mathrm{P}<0.001^{*}$ significantly different from PCOS treated with Metformin.

\section{Effect of Different Treatments on Biochemical Changes among the Groups}

Table 2 showed the comparison of different treatments on the rat's plasma level of lipids (mg/dl). In the PCOS induced group significant increase in plasma total cholesterol (TC), triglycerides (TG) $(\mathrm{P}<0.0001)$ while a highly significant decline in High-density lipoproteins (HDL) $(\mathrm{P}<0.0001)$ were observed as compared to the control group (group I). Non-significant changes were observed in the levels of LDL ( $\mathrm{P}>0.05)$.

Administration of aqueous extract of fenugreek seed (group III) showed a significant increase in plasma levels of HDL $(\mathrm{P}<0.0001)$ with a significant decrease in TC, TG $(\mathrm{P}<0.0001)$, and LDL showed a non-significant decrease in their values $(\mathrm{p}>0.05)$. Metformin group showed no significant effect on plasma LDL $(\mathrm{P}>0.05)$ while a significant effect was observed on TC, TG, HDL levels $(\mathrm{P}<0.0001)$.

Table 2: Effect of different treatments on biochemical changes among the groups

\begin{tabular}{|l|l|l|l|l|}
\hline GROUP & Parameters \\
\hline $\begin{array}{l}\text { Normal Control Group (Group } \\
\text { I) }\end{array}$ & CHOL (mg/dl) & TGs (mg/dl) & HDL (mg/dl) & LDL (mg/dl) \\
\hline $\begin{array}{l}\text { PCOS induced Group (Group } \\
\text { II }\end{array}$ & $115.4 \pm 5.97^{*}$ & $86.24 \pm 9.52^{*}$ & $32.14 \pm 1.79^{*}$ & $48.22 \pm 6.03^{* *}$ \\
\hline Fenugreek Group (Group III) & $74.47 \pm 0.550^{*}$ & $54.25 \pm 0.500^{*}$ & $62.95 \pm 1.10^{*}$ & $6.20 \pm 0.469^{* *}$ \\
\hline Metformin Group (Group IV) & $104.3 \pm 0.478^{*}$ & $65.30 \pm 0.476^{*}$ & $61.97 \pm 1.41^{*}$ & $44.90 \pm 62.73^{* *}$ \\
\hline
\end{tabular}

Values are presented as mean \pm S.D, applied one-way ANOVA followed by Tukey test $(\mathrm{n}=6)$.

$* \mathrm{P}<0.0001$ vs. control vs. letrozole vs. fenugreek seed vs. metformin (one-way ANOVA followed by TUKEY test)

** $p>0.005$ vs. control vs. letrozole vs. fenugreek seed vs. metformin (one-way ANOVA followed by TUKEY test)

\section{Comparison of Histological Examination of Ovarian Sections}

Figure 4 illustrates the histological examination of all groups of rats. Ovarian tissue sections showed that the normal Control group (Figure A) appears to have healthy follicles in various developmental stages with predominant corpus luteum while in the PCOS induced group (Figure B) many cystic follicles were observed. Some follicles were filled with secretion while no evidence of granuloma was found. Rats treated with metformin (Figure C) showed corpus luteum and follicle with multiple layers of granular cells. The fenugreek group (Figure D) showed improvement and decrease in the number of cysts and healthy corpus luteum as well. Figure E showed para tubular cyst of a fallopian tube in the PCOS induced group whereas F showed fallopian tubes of a normal control group. 
Biosight 2021; 02(02): 13-21
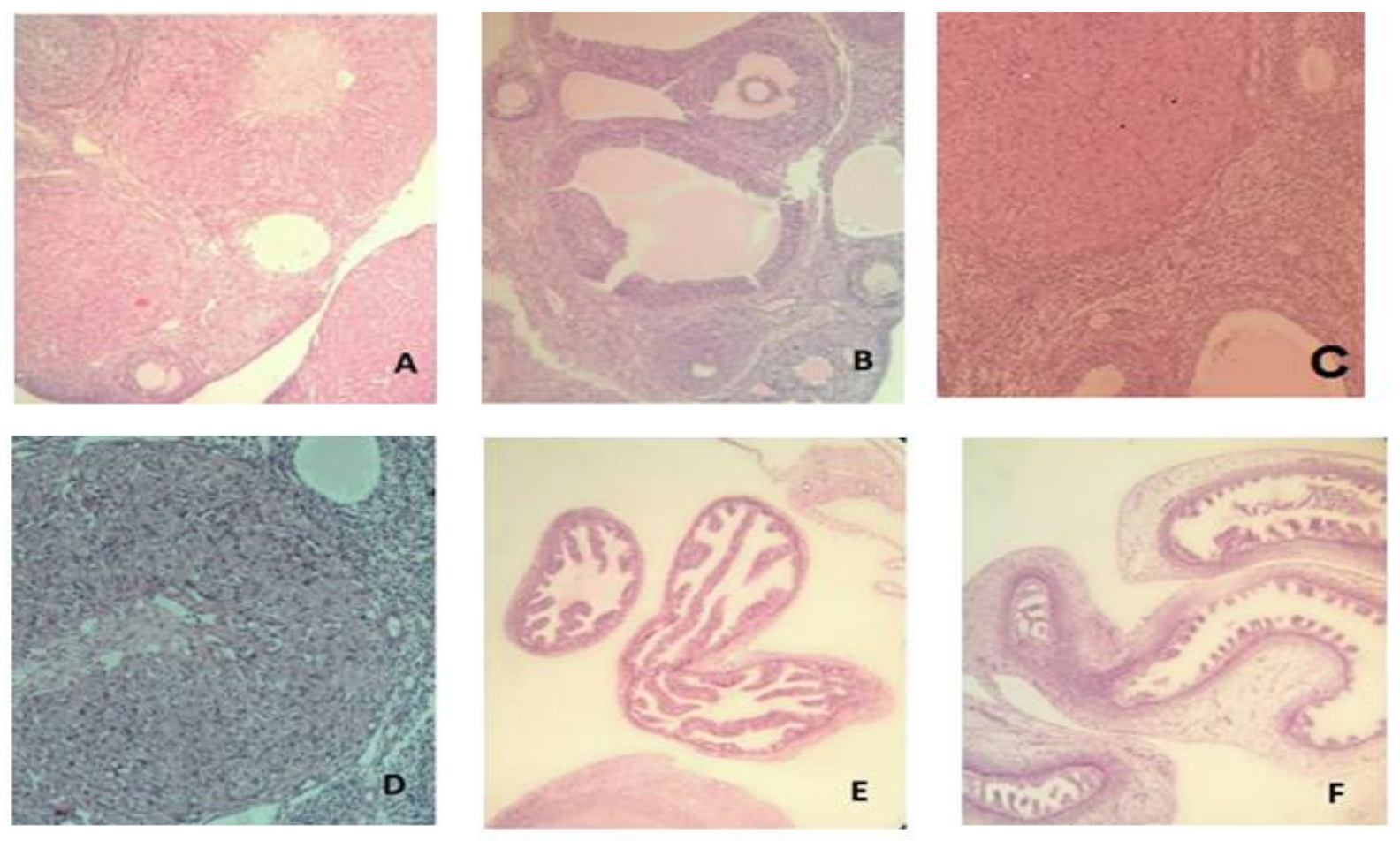

Figure 4: Photomicrograph of Transverse Histological Section of Ovary of different groups. (A)Showed Control Group With Corpus Luteum And Healthy follicle; (B)Showed PCOS induced Group With Cysts; (C) Metformin Group With Multi-Layer Granular Cells Follicle; (D) Fenugreek Group Showed Prominent Corpus Luteum And Small Occasional Cystic Follicle; (E) Showed Para tubal Cyst Of Fallopian Tube in PCOS induced group; (F) Fallopian Tube of normal control group.

\section{Discussion:}

Several animal models of PCOS have been developed over many decades. In the present study, letrozole (an aromatase inhibitor) was used to develop a rodent model and to investigate the therapeutic intervention of PCOS with the help of fenugreek seeds [12]. The results of our study showed that prepubertal exposure of Letrozole in female Wistar rats causes the development of ovarian cysts, hyperandrogenism, and hormonal disturbances which represents the clinical manifestation of human PCOS in many ways [13]. In the current years, clinical studies have reported the effectiveness of fenugreek seed on PCOS treatment [14]. This study was aimed to evaluate the hypoglycemic, anti-androgenic, and hypocholesterimc effect in PCOS-induced rats.

The induction of PCOS was confirmed by an irregular estrus cycle with persistent vaginal cornification and elevated levels of testosterone. The control group showed the normal estrous cycle throughout the whole experiment. PCOS-induced rats exhibited irregular estrous cycles along with a prolonged diestrus phase followed by an unknown phase, this phase is also called as pseudo-diestrus phase, in which few leukocytes were present but no other cells were seen $[15,16]$. Treatment of PCOS-induced rats with metformin and fenugreek seeds restored the normal estrous cycle in rats [17].

The changes in ovarian morphology include the appearance of polycystic ovaries with few corpora lutea in the PCOS-induced group as compared to the normal control group [17]. In Fenugreek treated group reduced no of cysts with different numbers of corporal lutea were observed which is an indicator of a normal estrus cycle [18]. PCOS-induced rats that were treated with metformin showed normal ovarian cortex and corpus luteum with a thick layer of granulosa cells [19].

Moreover, PCOS induced group showed an increase in their body weight compared to the control group. In treatment groups, the weight of PCOS-induced rats was restored to their normal levels [20, 21]. 
PCOS is associated with multiple hormonal and metabolic derangements manifested in the form of hyperglycemia, insulin resistance, and resultant hyperinsulinemia. Many studies have reported the induction of hyperglycemia in Letrozole-induced PCOS animal models [22]. Our study also found elevated glucose levels in PCOS-induced rats as compared to the control group. The treated groups (Fenugreek and Metformin) have been shown to cause hypoglycemia which is incongruity with the previous findings. In the present study, insulin resistance with concomitant hyperinsulinemia was observed in the PCOS-induced group with respect to the normal control group. PCOS-induced rats treated with metformin and fenugreek group showed a significant decrease in insulin levels as compared to the PCOS-induced group [19] (Table 1).

Furthermore, PCOS-induced rats had elevated testosterone levels as compared to the control group because letrozole blocks the conversion of androgen substrate into estrogen [23]. Both fenugreek seed extract and metformin assisted in the reduction of testosterone levels in PCOS rats which is in agreement with earlier findings [19, 24].

Several studies have reported the dyslipidemic effect of Letrozole which might be a consequence of hyperandrogenism (Table 2) [23]. The results of our study showed elevated plasma lipids (TC, TGs,) and low levels of HDL and LDL than the control group. Fenugreek extract administration resulted in decreased TC, TGs levels whereas it did not have a profound effect on plasma HDL and LDL levels. Literature reveals that some active components of fenugreek like saponin and galactomannan are involved in reducing lipids except HDL (25). Metformin treated group showed a significant change in TC, TG, and HDL like the control group except for LDL which shows the non-significant difference $(\mathrm{P}>0.05)[17,23]$.

\section{Conclusion:}

We concluded from the current study that letrozole served as the convenient model of PCOS. The aqueous extract of fenugreek seeds possesses hypoglycemic, hypoandrogenic, and hypocholesterologenic effects. Fenugreek could serve as an anti-infertility therapeutic agent by preventing hormonal disturbances, ovarian cell dysfunction and restoring the normal ovarian architecture. Thus, due to the broad-spectrum effects of fenugreek seeds, it might be used in the cure of endocrine and ovarian derangements in PCOS.

\section{List of Abbreviations}

PCOS: Polycystic ovary syndrome,

T: Testosterone,

TC: Total cholesterol,

TGs: Triglycerides,

LDL: Low density lipoprotein,

HDL: High density lipoprotein.

\section{Authors' Contributions}

Uzma Firdous conceived and designed the study and analyzed the data, Bushra Manzoor Lodhi performed all the experiments, did a literature survey, and drafted the manuscript.

\section{ETHICS APPROVAL AND CONSENT TO PARTICIPATE}

Not applicable.

\section{HUMAN AND ANIMAL RIGHTS}

No animals were used in this study. The study on humans was conducted in accordance with the ethical rules of the Helsinki Declaration and Good Clinical Practice.

\section{CONSENT FOR PUBLICATION}

Not applicable. 
Biosight 2021; 02(02): 13-21

\section{AVAILABILITY OF DATA AND MATERIALS}

None.

\section{FUNDING}

None.

\section{CONFLICT OF INTEREST}

The authors declare no conflict of interest, financial or otherwise.

\section{ACKNOWLEDGEMENTS}

None.

\section{REFERENCES}

1. Stener-Victorin E, Padmanabhan V, Walters KA, Campbell RE, Benrick A, Giacobini P, et al. Animal models to understand the etiology and pathophysiology of polycystic ovary syndrome. Endocrine Rev 2020; 41(4): 538-76.

2. Anwar N, Hamid S, Nadeem A, Asad A, Waseem N, Mehmood N. Effect of nigella sativa on serum testosterone levels in letrozole induced polycystic ovarian syndrome in mice. PAFMJ 2021; 71(1): 338-42.

3. $\quad$ Eisenberg ME. MD Polycystic ovary syndrome (pcos) fact sheet. 2010.

4. Hanem LGE, Stridsklev S, Júlíusson PB, Salvesen $\emptyset$, Roelants M, Carlsen SM, et al. Metformin Use in PCOS Pregnancies Increases the Risk of Offspring Overweight at 4 Years of Age: Follow-Up of Two RCTs. J Clin Endocrinol Metabol 2018; 103(4): 1612-21.

5. Viollet B, Guigas B, Garcia NS, Leclerc J, Foretz M, Andreelli F. Cellular and molecular mechanisms of metformin: an overview. Clin Sci 2012; 122(6): 253-70.

6. Dujic T, Causevic A, Bego T, Malenica M, Velija-Asimi Z, Pearson E, et al. Organic cation transporter 1 variants and gastrointestinal side effects of metformin in patients with Type 2 diabetes. Diabet Med 2016; 33(4): 511-4.

7. Wang $\mathrm{J}, \mathrm{Wu} \mathrm{D}$, Guo $\mathrm{H}, \mathrm{Li} \mathrm{M}$. Hyperandrogenemia and insulin resistance: The chief culprit of polycystic ovary syndrome. Life Sci 2019; 236: 116940.

8. Ahmad A, Alghamdi SS, Mahmood K, Afzal M. Fenugreek a multipurpose crop: Potentialities and improvements. Saudi J Biol Sci 2016; 23(2): 300-10.

9. Sayyah-Melli M, Mobasseri M, Gharabaghi P, Ouladsahebmadarek E, Rahmani V. Comparing the effect of aromatase inhibitor (letrozole)+ cabergoline (Dostinex) and letrozole alone on uterine myoma regression, a randomized clinical trial. Eur J Obstetric Gynecol Reproductive Biol 2017; 210: 257-64.

10. Noor EA. Comparative study on the corrosion inhibition of mild steel by aqueous extract of Fenugreek seeds and leaves in acidic solutions. J Eng Appl Sci 2008; 3(1): 23-30.

11. Byers SL, Wiles MV, Dunn SL, Taft RA. Mouse estrous cycle identification tool and images. PloS one 2012; 7(4): e35538.

12. Kim EJ, Jang M, Choi JH, Park KS, Cho IH. An improved dehydroepiandrosterone-induced rat model of polycystic ovary syndrome (PCOS): Post-pubertal Improve PCOS's features. Front Endocrinol 2018; 9(735).

13. Yu J, Ding C, Hua Z, Jiang X, Wang C. Protective effects of berberine in a rat model of polycystic ovary syndrome mediated via the PI3K/AKT pathway. J Obstetric Gynaecol Res 2021.

14. Rao SK. An insight on polycystic Ovary syndrome (PCOS) and use of herbal medicines as alternative treatment. Treating endocrine and metabolic disorders with herbal medicines: IGI Global 2021; p. 125-63.

15. Rajan RK, Balaji B. Soy isoflavones exert beneficial effects on letrozole-induced rat polycystic ovary syndrome (PCOS) model through anti-androgenic mechanism. Pharm Biol 2017; 55(1): 242-51.

16. Yang H, Lee SY, Lee SR, Pyun B-J, Kim HJ, Lee YH, et al. Therapeutic effect of ecklonia cava extract in letrozole-induced polycystic ovary syndrome rats. Front Pharmacol 2018; 9(1325).

17. Osman NN, Alsahfi SA, Alshubaily F. Effectiveness of aqueous extract of fenugreek seeds and flaxseed on polycystic ovarian syndrome in female rats. Int J Pharm Res Allied Sci 2019;8(4):42-54. 
Biosight 2021; 02(02): 13-21

18. Swaroop A, Jaipuriar AS, Gupta SK, Bagchi M, Kumar P, Preuss HG, et al. Efficacy of a novel fenugreek seed extract (Trigonella foenum-graecum, FurocystTM) in polycystic ovary syndrome (PCOS). Int J Med Sci 2015; 12(10): 825.

19. Zhang S, Tu H, Yao J, Le J, Jiang Z, Tang Q, et al. Combined use of diane-35 and metformin improves the ovulation in the PCOS rat model possibly via regulating glycolysis pathway. Reproduct Biol Endocrinol 2020; 18: 1-11.

20. Hassanzadeh Bashtian M, Emami SA, Mousavifar N, Esmaily HA, Mahmoudi M, Mohammad Poor AH. Evaluation of fenugreek (Trigonella foenum-graceum L.), Effects seeds extract on insulin resistance in women with polycystic ovarian syndrome. Iran J Pharm Res 2013; 12(2): 475-81.

21. Kristensen M, Jensen MG, Aarestrup J, Petersen KE, Søndergaard L, Mikkelsen MS, et al. Flaxseed dietary fibers lower cholesterol and increase fecal fat excretion, but magnitude of effect depend on food type. Nut Metabol 2012; 9(1): 1-8.

22. Zayed EA, AinShoka AA, El Shazly KA, El-Mosallamy AE, Zayed AA, Abd El Latif HA. Fenugreek oil and metformin improve insulin resistance via increase of GLUT4 and PPAR $\gamma$ in metabolic syndrome-induced rats. Asian J Res Rep Endocrinol 2021: 29-39.

23. Alkalby JM, Hamzah FZ. Ameliorative Effect of fenugreek on sex hormones in polycystic ovary syndrome female rats induced by letrozole. Kufa J Veterin Med Sci 2017; 8(2): 24-32.

24. Alkalby JM, Hamzah FZ. Ameliorative effect of fenugreek on sex hormones in polycystic ovary syndrome female rats induced by letrozole. Kufa J Veterin Med Sci 2017; 8(2).

25. Kassaian N, Azadbakht L, Forghani B, Amini M. Effect of fenugreek seeds on blood glucose and lipid profiles in type 2 diabetic patients. Int J Vitamin Nut Res 2009; 79(1): 34-9. 